\author{
Military Technical College \\ Kobry Elkobbah, \\ Cairo, Egypt
}

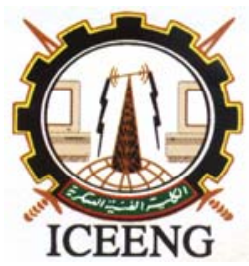

\author{
$5^{\text {th }}$ International Conference \\ on Electrical Engineering \\ ICEENG 2006
}

\title{
MULTIUSER CHANNEL ESTIMATION AND DEECTION FOR WCDMA SYSTEMS
}

\author{
A. El-Bardaweny ${ }^{*}$, A. El-Mahdy ${ }^{*}$ E. Saad ${ }^{* *}$
}

\begin{abstract}
This paper focuses on the design of a multi-user receiver structure for the reverse link of a Wideband Code-Division Multiple-Access (WCDMA) communication system. The detection is based on a ML-based channel estimation algorithm. Once a composite Channel Impulse Response (CIR) of each user is estimated, it is directly used in the detection process instead of first extracting the individual channel parameters, such as path delays and attenuation factors. The paper presents a framework that facilitates a computationally efficient solution to the combined problem of channel estimation and detection in a multiuser multi-path environment. The performance of the different detectors is evaluated in terms of bit error probability using the estimated composite CIR.
\end{abstract}

\section{KEYWORDS}

Multi-user detection, estimation, WCDMA

\section{INTRODUCTION}

Code Division Multiple Access (CDMA) is the multiple access technology selected for today's standards of third generation wireless communications [1]. Several standards for third generation systems have been proposed and developed by different industrial countries such as U.S, Europe, and Japan. All these standards have accepted CDMA in one form or another as the multiple access method for wireless communications.

One of the main problems in the base-station receiver is the estimation and detection of transmitted data from the received signal. Channel parameter estimation is essential, particularly when the mobile system is subject to multi-path fading, as in the WCDMA that is, the transmission channel consists of more than one distinct propagation path for each user's signal. The goal of channel parameter estimation is to determine the time varying attenuation factors and the delays of the different paths by processing the received signal, in order to facilitate recovery of the data transmitted by each user.

\footnotetext{
*Egyptian Armed Forces, **Sudanese Armed Forces.
} 
When studying the different algorithms for the estimation of the channel complex coefficients, most authors usually assume that perfect knowledge about the path delays is coefficients available at the receiver, however, in a realistic CDMA receiver both channel coefficient estimation and delay estimation tasks should be performed, either in a joint or in a decoupled manner.

The front-end of the receiver is the demodulator and chip matched filter, it retrieves the base-band signal for further processing. The continuous time base-band signal from the channel is converted to a discrete time signal by sampling the output of a filter matched to the chip waveform. The chip matched filtering is an integration and dump of the base-band signal correlated with the chip waveform, over the chip duration, with the output held at the end of the chip duration.

Due to the asynchronous nature of the transmissions and due to the path delays introduced by the channel, the base-band signal corresponding to the bit-streams of different users is received at different delayed instants in time. The multi-user detector needs the knowledge of the bit boundaries of all the users for detection and interference suppression. Certain kinds of detectors also need the amplitude and phase information of each user [2]. The channel estimation procedure gives the arbitrary delay, the magnitude and phase change introduced by the channel for all the users. The initial part of the channel estimation involves an acquisition phase, where the first estimate of the parameters is obtained. For the rest of the transmission, it involves tracking, where the channel parameters are continuously updated. Tracking is required for a dynamic channel, where the properties of the channel change with time due to multi-path fading effects.

The detection process retrieves the bits of a particular user from the superimposed baseband signal, which has the chip streams of different users. The detector needs the knowledge of the spreading sequence of a user to detect the bits. The base-station receiver uses a multi-user detection scheme, where the bits of all the users are detected.

The paper is organized into the following sections. Section 2 presents the WCDMA system under study and a model for the multi-path channel. In section 3 we describe the maximum likelihood channel estimation algorithm. Section 4 discuses the multi-user detection and section 5 presents the results of the conducted simulations. The main conclusions and directions for future research are presented in section 6 .

\section{SYSTEM MODEL}

In this section, a system model that handless the effect of multi-path on the different users efficiently is developed. The model doesn't assume chip synchronization. $K$ active users using direct sequence CDMA system with Binary Phase Shift Keying (BPSK) modulation are assumed. Each user transmits a zero mean stationary bit sequence with independent identically distributed components and the different users are independent of each other.

The complex base-band $k^{\text {th }}$ user's transmitted signal is 


$$
s_{k}(t)=\sqrt{E_{k}} \sum_{i=1}^{L} b_{k, i} c_{k}(t-i T)
$$

where $E_{k}$ is the transmitted power, $b_{k, i} \in\{+1,-1\}$ is the $i^{\text {th }}$ transmitted bit and $c_{k}(t)$ is the spreading waveform. The spreading code waveform is composed of $N$ chips and if BPSK is assumed for the spreading modulation, then $c_{k}(t)=\sum_{n=0}^{N-1} c_{k, n} \Pi\left(t-n T_{c}\right)$, where $c_{k, n} \in\{+1,-1\}$ and the chip pulse waveform $\Pi(t)$ is a rectangular pulse of duration $T_{\mathrm{c}}$. Short code spreading is assumed and hence $T=N T_{c}$, where $T$ is the symbol period.

The channel impulse response $h_{k}(t)$ seen by user $k$ is

$$
h_{k}(t)=\sum_{p=1}^{P} \omega_{k, p} \partial\left(t-\tau_{k, p}\right)
$$

where, $\omega_{k, p}$ is the complex amplitude of the received $p^{\text {th }}$ path of the $k^{\text {th }}$ user and $\tau_{k, p}$ is the relative delay with respect to a reference at the receiver. The delay spread is assumed to be less than half the symbol period. The channel parameters are assumed to be unknown but remain static during the estimation time. Accordingly, the received signal at the base station is

$$
r(t)=\sum_{k=1}^{K} \sum_{p=1}^{P} \omega_{k, p} S_{k}\left(t-\tau_{k, p}\right)+v(t)
$$

the noise component $v(t)$ is assumed to be Gaussian with zero-mean and double sided spectral density of $N_{o} / 2$.

The observation vector $\mathbf{r}_{i}{ }^{\text {TM }} \mathrm{X}^{N}$, is formed by collecting $N$ successive outputs $r(n)$, from a chip-matched filter sampled at the chip rate [3]. Hence,

$$
\mathbf{r}_{i}=[r(i), r(i+1), \ldots, r(i+N-1)]^{T}
$$

The receiver has an arbitrary timing reference that will not be aligned to actual transmitted bit boundaries. Hence each observation vector can be viewed as a linear combination of $2 K$ signal vectors, two components from each user, as shown in Fig.1. 


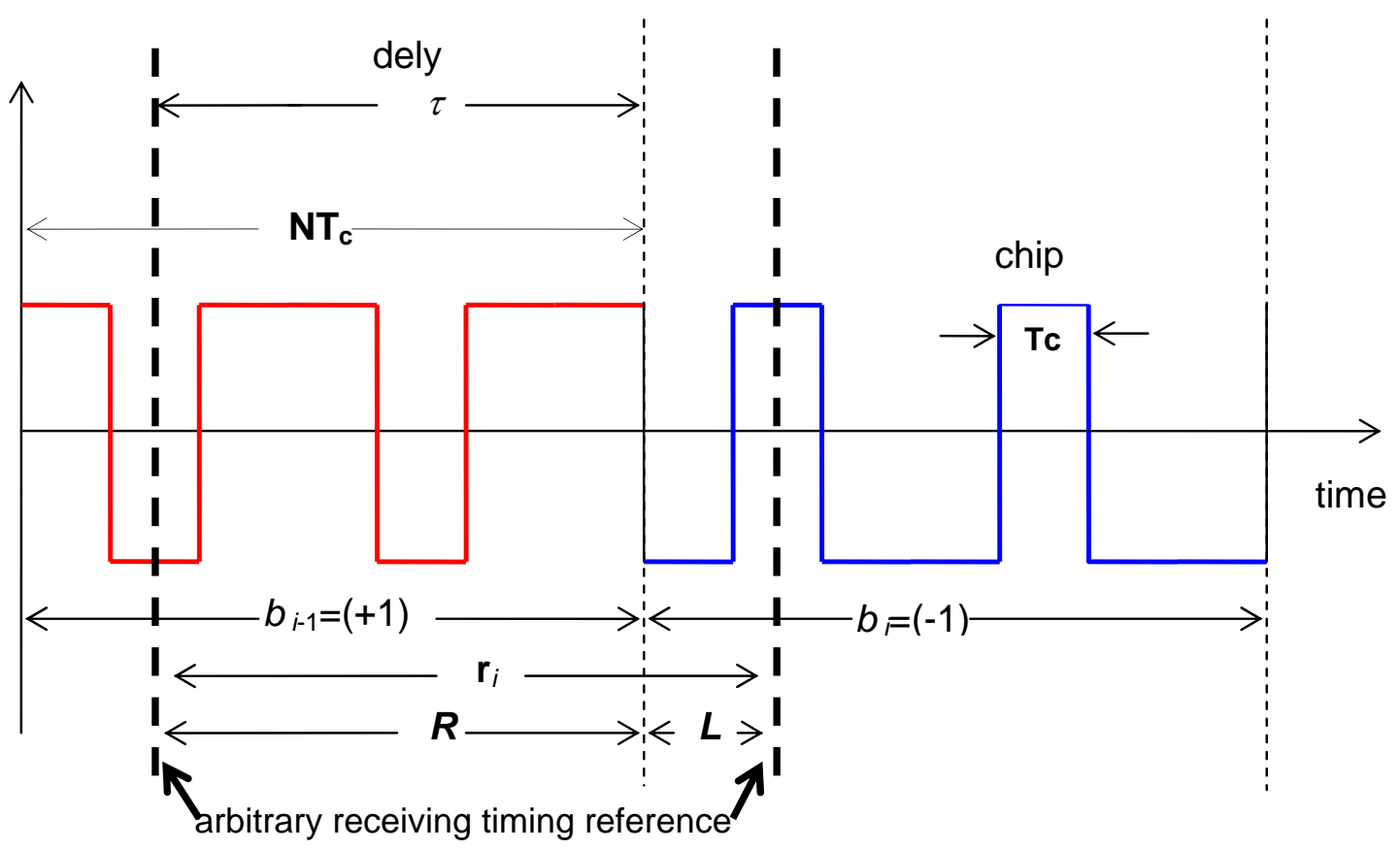

Figure 1: System model-received signal

The contribution of the spreading code of the user to the vector $\mathbf{r}_{i}$ appears in two parts, the right part (shown as $R$ ) of bit $\mathbf{b}_{i-1}$ and the left part (shown as $L$ ) of bit $\mathbf{b}_{i}$.Now $\mathbf{r}_{i}$ can be written as :

$$
\mathbf{r}_{i}=\mathbf{U Z b}_{i}+\boldsymbol{v}_{\boldsymbol{i}} ; \quad \boldsymbol{v}_{\boldsymbol{i}} \sim N(\mathbf{0}, \mathbf{K})
$$

where $\mathbf{U}$ is a known matrix of spreading codes and $\mathbf{Z}$ has all the unknown parameters of all users, $\mathbf{b}_{i}$ contain the users' data bits given by

$$
\mathbf{b}_{i}=\left[b_{1, i-1}, b_{1, i}, \ldots, b_{K, i-1}, b_{K, i}\right]^{T}
$$

and $\mathbf{K}$ is the unknown noise covariance.

\section{MULTIUSER CHANNEL ESTIMATION}

Multi-user Channel estimation refers to the joint estimation of amplitudes and delays for all active users. The channel parameters to be extracted from the information contained in the received pilot signal. Since the observation vector $\mathbf{r}_{i}$ depends on the channel whose a priori statistics are unknown, a maximum likelihood estimate of the channel is often used. In our problem, $\mathbf{r}_{i}$ is a function of the channel vector $\mathbf{z}_{k}$, the noise covariance matrix $\mathbf{K}$, which is assumed unknown and the transmitted bits $\mathbf{b}_{i}$. We assume the bits $\mathbf{b}_{i}$ are known. 
This accomplished in the acquisition phase by requiring that all users transmit training pilot sequences. The discussion here refers to the maximum likelihood based channel estimation scheme [4]. In this model, it is assumed that the maximum delay spread between the paths is less than half a symbol period.

The following steps occur in the maximum likelihood estimation method:

$$
\begin{aligned}
& \hat{\mathbf{R}}_{r r}=\frac{1}{L} \sum_{i=1}^{L} \mathbf{r}_{i} \mathbf{r}_{i}^{H}, \\
& \hat{\mathbf{R}}_{b r}=\frac{1}{L} \sum_{i=1}^{L} \mathbf{b}_{i} \mathbf{r}_{i}^{H}, \\
& \hat{\mathbf{R}}_{b b}=\frac{1}{L} \sum_{i=1}^{L} \mathbf{b}_{i} \mathbf{b}_{i}^{T}
\end{aligned}
$$

where is $\hat{\mathbf{R}}_{r}$ the autocorrelation of the observation vector, $\hat{\mathbf{R}}_{b r}$ the cross correlation between the observation vector and the preamble, $\mathbf{R}_{b b}$ the autocorrelation of the preamble bits, $\mathbf{K}$ the noise covariance matrix, the estimate of $\mathbf{U Z}, \mathbf{U}$ the matrix of codes and $\mathbf{Z}$ the channel impulse response matrix. The ML estimate of $\Psi=\mathbf{U Z}$ is shown to be:

$$
\hat{y}=\hat{\mathbf{R}}_{r b} \hat{\mathbf{R}}_{b b}^{-1}
$$

the noise covariance is

$$
\hat{\mathbf{K}}(\hat{y})=\hat{\mathbf{R}}_{r}-\hat{y} \hat{\mathbf{R}}_{r}^{H}
$$

and the closed-form expression for the composite CIR vector $\hat{\mathbf{z}}_{k}$ for each user is

$$
\hat{\mathbf{z}}_{k}{ }^{H}=\left(\hat{\mathbf{y}}_{2 k-1} \hat{\mathbf{K}}^{-1} \mathbf{U}_{k}^{R}+\hat{\mathbf{y}}_{2 k} \hat{\mathbf{K}}^{-1} \mathbf{U}_{k}^{L}\right)\left(\mathbf{U}_{k}^{R^{H}} \hat{\mathbf{K}}^{-1} \mathbf{U}_{k}^{R}+\mathbf{U}_{k}^{L^{H}} \hat{\mathbf{K}}^{-1} \mathbf{U}_{k}^{L}\right)^{-1}
$$

\section{MULTI-USER DETECTION}

In this section, we explore the use of the composite CIR, in the conventional detector and in a linear multi-user detector. We propose to use the CIR vector directly in the detection processes, instead of first extracting individual parameters.

\section{Matched Filter Detector}

The conventional detector for the received signal in a single path environment is a bank of $K$ code-matched filters. For the $k^{\text {th }}$ user, the matched filter, matched to the single path component, is usually implemented using channel parameters as:

$$
\mathrm{y}_{k}(i)=\int_{i T}^{(i+1) T} \omega_{k}^{*} r(t) C_{k}\left(t-i T-\tau_{k}\right) d t
$$


where $\mathrm{y}_{k}(i)$ is the matched filter output for the $k^{\text {th }}$ user at the $i^{\text {th }}$ time step, (.) * denotes the complex conjugate of (.). The outputs of the matched filters yield soft estimates of the transmitted bits. The final hard decisions are the sign of these soft estimates.

Using the discretized version of the received signal model (6), the equation for the matched filter output, for the multi-user and multi-path, is expressed in vector form as:

$$
\mathbf{y}_{i}=(\mathbf{U Z})^{H} \mathbf{r}_{i}
$$

In this model, equation (13) is similar to the notation of a received effective signature waveform, described in [5]. The received effective signature waveform is the spreading code of a user modified with the corresponding channel parameters, which is exactly the result of ( $\mathbf{U Z}$ ), for all the users. Since all the delay is captured in $\mathbf{Z}$, (13) can be interpreted as a full code-matched filter operation. This matched filter would be optimum for a single user only, in an Additive White Gaussian noise (AWGN) channel.

Closer inspection of (13) reveals that we can carry this process one step further. The expression for the received signal and hence the detectors consist of the term $u_{k}^{R} \mathbf{z}_{k}$ or $u_{k}^{L} \mathbf{z}_{k}$, instead of $\mathbf{z}_{k}$ in isolation. Actually, the terms $u_{k}^{R} \mathbf{z}_{k}$ or $u_{k}^{L} \mathbf{z}_{k}$ are the spreading code of a user modified with the corresponding channel parameters or the received effective signature waveform. These are the two terms of primary interest to the detector and not the individual channel parameters or even the composite response $\mathbf{z}_{k}$.

So, instead of estimating individual parameters or the composite response $\mathbf{z}_{k}$, it is sufficient if the channel estimation step estimates $u_{k}^{R} \mathbf{z}_{k}$ and $u_{k}^{L} \mathbf{z}_{k}$. It may be recalled from [4], that these two terms are the columns of matrix $\Psi$, the estimation of which is the first step (9) in the maximum likelihood algorithm. Using the developed algorithm to estimate is quite straightforward, and saves computation when compared to estimating $\mathbf{z}_{k}$ or the individual parameters. The savings in computation comes from reducing the channel estimation algorithm to computation of the sample correlation matrices followed by computation of the effective spreading code. The performance will also improve as the error from the estimation of $\mathbf{z}_{k}$ from $\mathbf{U Z}$ and extraction of the individual parameters can be avoided.

\section{Decorrelator Detector}

The idea of using the composite channel impulse response vector in detection can also be applied to linear multi-user detectors. Linear multi-user detectors apply a linear mapping $G$, to the soft output of the matched filters to reduce the multiple access interference seen by each user.

Therefore, the vector of decision statistics for all the users, from a linear single-shot detector, $G \in \square^{2 K \times 2 K}$, is given by : 


$$
\mathbf{x}_{i}=G \mathbf{y}_{i}=G(U \mathbf{Z})^{H} \mathbf{r}_{i}
$$

The required bit estimates are the hard decision $\hat{\mathbf{b}}_{i}=\operatorname{sign}\left(\operatorname{real}\left(\mathbf{x}_{i}\right)\right)$. According to our system model, $\mathbf{b}_{i}$ contains consecutive bits of each user. Hence the decision statistic for each user will consist of two soft estimates for each bit of the user. The two soft estimates can be combined such that the output signal-to-interference ratio is maximized, to obtain a hard decision for each bit.

The decorrelating detector $G_{\text {dec }}$ provides the maximum likelihood linear estimate of the received bits, given the output of the code-matched filters [6]. Hence, it follows that:

$$
G_{d e c}=\left((U \mathbf{Z})^{H}(U \mathbf{Z})\right)^{-1}
$$

In the following section, we will report results of simulations that show the performance gains obtained from the detectors, using the composite channel impulse response vector.

\section{SIMULATION RESULTS}

In this section, we describe the simulations that we conducted to evaluate the performance of the different detectors using the composite channel impulse response provided by the estimator, in the multi-path case. Experiments are conducted using Matlab ${ }^{\circledR}$ programming language.

Gold codes of length $N=31$ were used as spreading codes in all the simulations. The delays of all the users were assumed uniformly distributed in $[1 ; 31)$ chips. The Multiple Access Interference (MAI) presented by each interferer, which is the ratio of the interferer's and desired user's received energies, was uniformly distributed in $[0 ; M A I] \mathrm{dB}$. The number of paths for each user is denoted by $P$, the number of observations by $L$, the number of users by $K$, and the Signal-to-Noise Ratio of the background noise is SNR.

The performance of the ML estimator, in terms of mean squared error, resistance to MAI and loss due to estimation was shown in [4].

The performance of a code-matched filter detector for different numbers of users is shown in Fig. 2. It is clear that the bit error probability obtained from using the known $\mathbf{U Z}$ matrix is almost the same as that obtained using the estimated UZ.

Fig.3 shows the bit error probability obtained from the matched filter receiver as the SNR is increased. The results show that the performance of the detector under different situations of composite CIR. First, the channel impulse response of the desired user is assumed to be known in form of $\mathbf{U Z}$ matrix. Second it is estimated using the algorithm, and the estimate of $\mathbf{U Z}$ is used in the detection. Third the estimate of $\mathbf{z}_{k}$ of the individual user is used directly in the detection. It is clear that the error probability from using the estimate of the matrix $\mathbf{U Z}$, is exactly the same as the error probability from using the known matrix $\mathbf{U Z}$. However the error probability from using $\mathbf{z}_{k}$ is slightly higher, due to the error introduced by the further computations of the estimate. 
The performance of the decorrelator detector is shown if Fig.4 for a ten user system using the known and estimated composite CIR (UZ).

\section{CONCLUSIONS}

The paper presents a framework that facilitates an efficient solution to the combined problem of multi-path channel estimation and detection for a set of transmitting users in the reverse link of a WCDMA communication system. We have shown that the estimated composite impulse response can be used directly in the detection, in a form of the matrix $\mathbf{U Z}$, without need for calculating the individual $\mathbf{z}_{k}$. The performance of the combined channel estimation and detection is illustrated in terms of BER for different cases. More investigations in the future are needed to consider other types of linear detectors, and possibility of extension to an antenna array system.

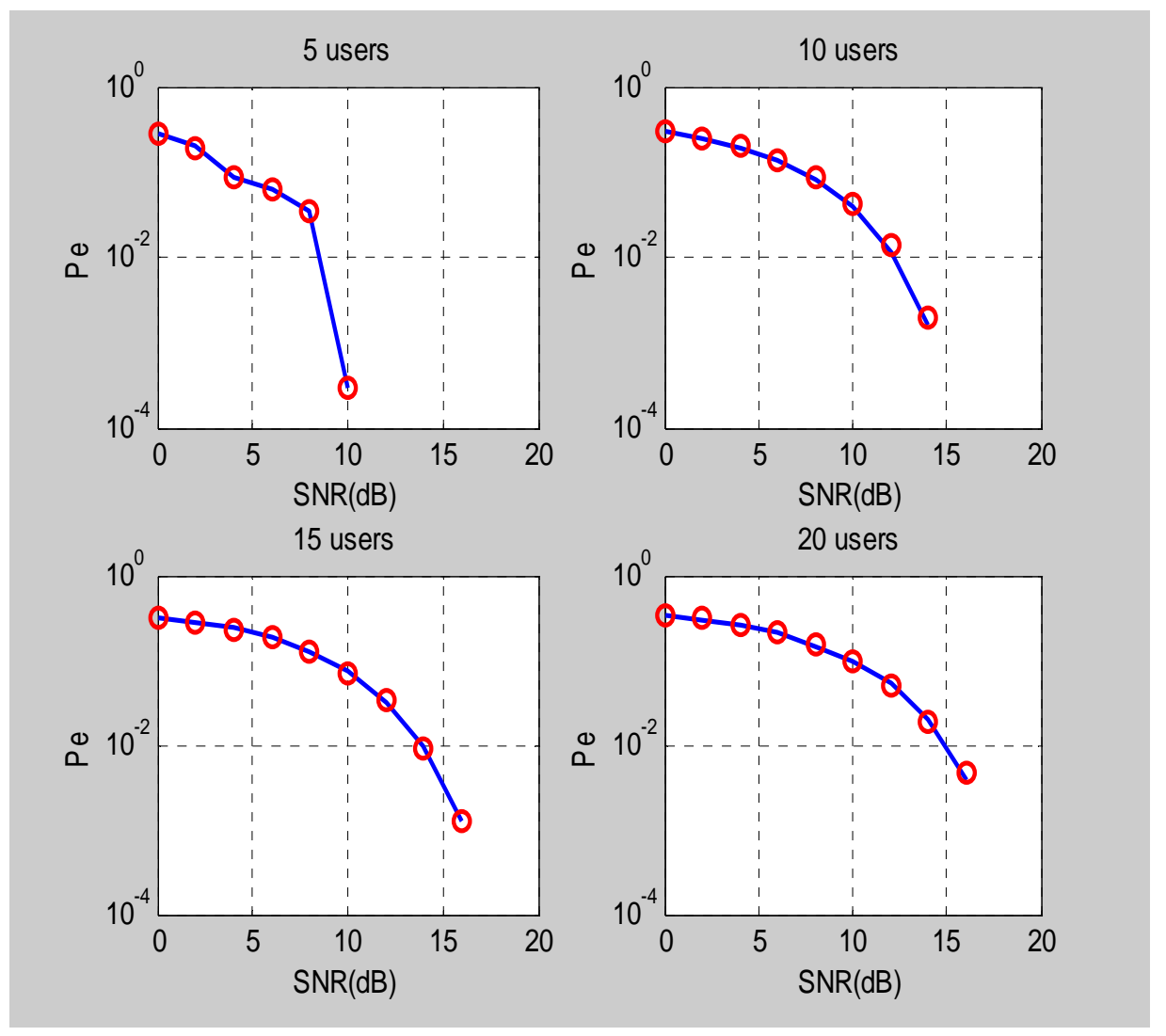

Fig.2 Probability of error versus SNR of a code-matched filter detector for different numbers of users using the known $\mathbf{U Z}$ shown as solid lines and estimated $\mathbf{U Z}$ shown as circles. 


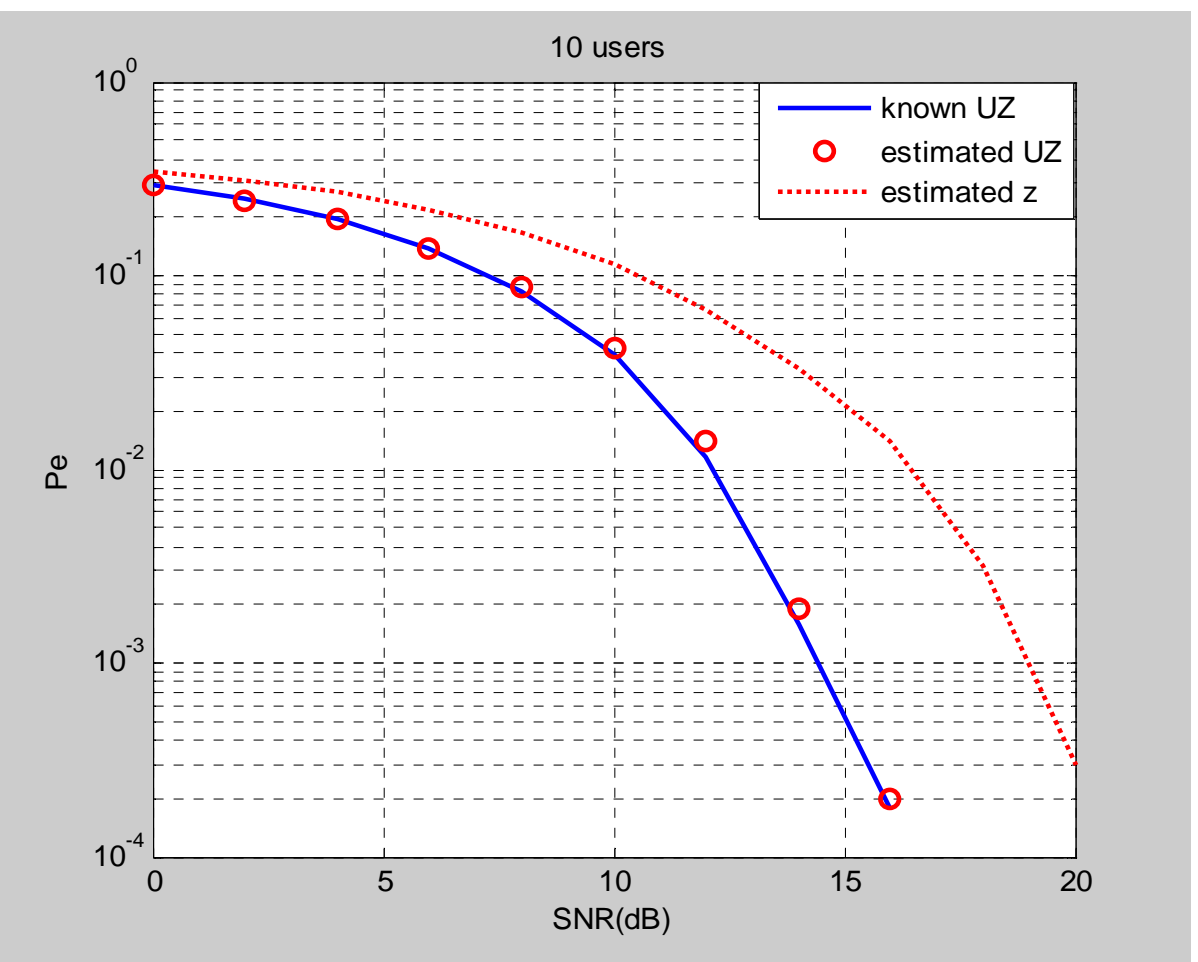

Fig.3 BER from a code-matched receiver using known $\mathbf{U Z}$, estimated $\mathbf{U Z}$ and estimated $\mathbf{z}_{k}$

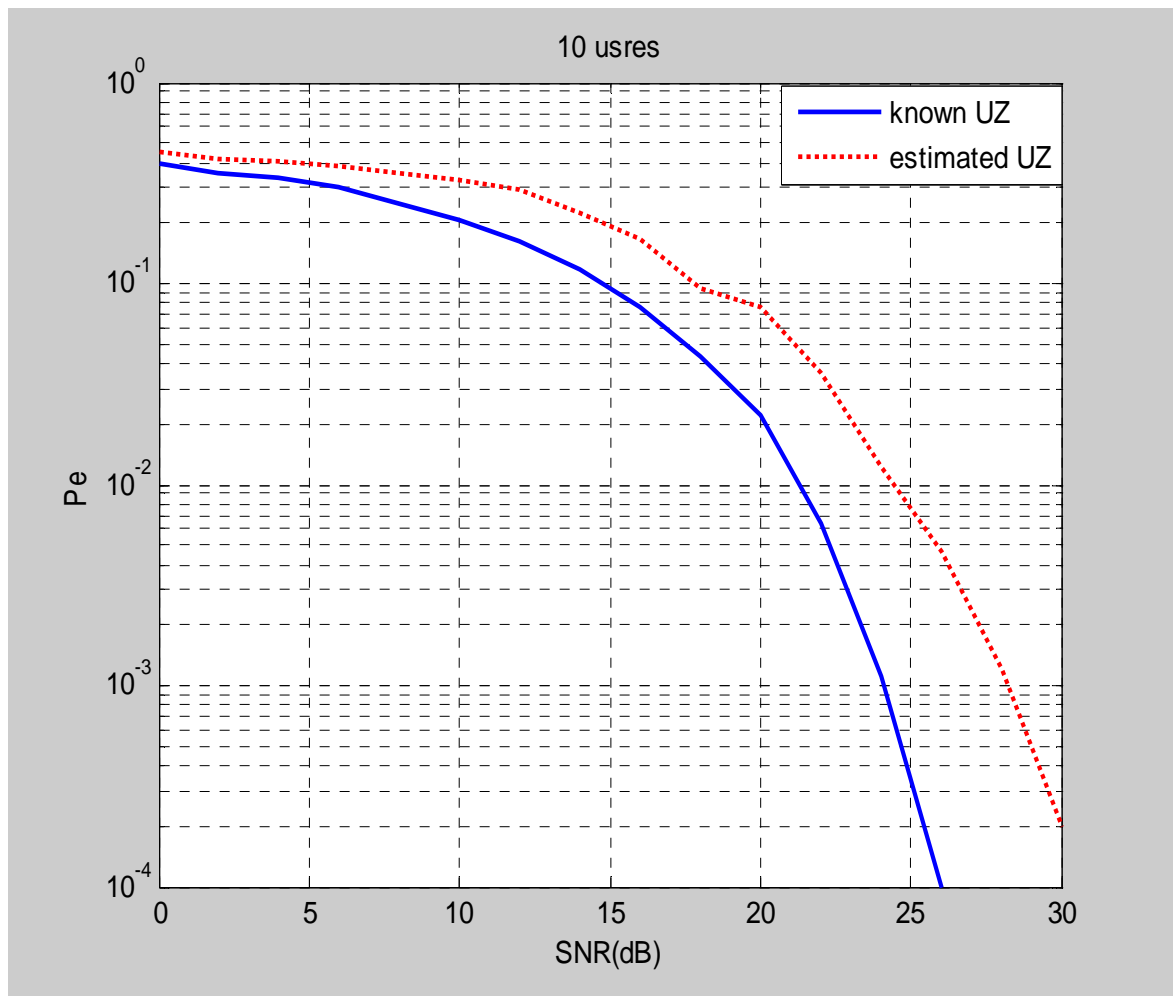

Fig.4 Probability of error $(\mathrm{Pe})$ versus $S N R$ for a decorrelator detector using the known composite CIR compared to the estimated composite CIR. 


\section{REFERENCES}

[1] 3GPP. Physical layer-general description. Technical Report TS 25.201 V3.0.0, available via web, at http://www.3gpp.org/ftp/Specs/latest/R1999/ (active in Sep 2003).

[2] Sergeo Verdu, Multiuser Detection,Cambridge University Presss,1998.

[3] Simon Haykin and Michael Moher, Modern Wireless Communications, Pearson Education Inc. Upper Saddle River 2005, NJ 07458.

[4] A. El-Bardaweny, K. El-Barbary. A. El-Mahdy and E. Saad. "Efficient Channel Estimation for WCDMA Systems", accepted for publication in the proceedings of the 5th ICEENG. M.T.C, Cairo 16-18 May. 2006

[5] X.Wang and H. V. Poor ,"Subspase-based blind joint interference suppression and channel estimation in multi-path CDMA channels," in Proc.IEEE Int. Conf. Universal Personal Communications(ICUPC), San Diego, CA, Oct.1997, pp 460464

[6] R. Lupas and S. Verd'u. "Linear multiuser detectors for synchronous code division multiple access channels". IEEE Trans. Information Theory, IT-35(123136), January 1989. 